Particle Accelerators, 1988, Vol. 24 pp. 1-10

Reprints available directly from the publisher

Photocopying permitted by license only

(C) 1988 Gordon and Breach Science Publishers, Inc.

Printed in the United States of America

\title{
END EFFECTS IN QUANTUM BEAMSTRAHLUNG
}

\author{
M. BELL and J. S. BELL \\ CERN, Geneva 23, Switzerland
}

(Received May 2, 1988)

End effects in quantum beamstrahlung, in the case of a uniform deflecting field, in the extreme quantum limit, are calculated. The result is applied to beamstrahlung with uniform cylindrical bunches and small disruption.

\section{INTRODUCTION}

The quantum theory of beamstrahlung energy loss for highly relativistic particles in quasiclassical orbits was developed by Schwinger ${ }^{1}$ and a series of Soviet theorists. ${ }^{2-6}$ In particular, the case of a uniform deflecting field was worked out in detail. In application to the beamstrahlung problem in linear colliders, ${ }^{7,8}$ the field was treated as locally uniform, and the results were averaged over the various strengths present in the bunch. The question arose as to on what scale the field must be approximately uniform for this treatment to be valid.

When the beamstrahlung process is largely classical, the important characteristic length is

$$
L_{C}=\rho / \gamma,
$$

where $\rho$ is the radius of curvature of the track, and $\gamma$ is the usual relativistic Fitzgerald contraction factor. A condition for the process to be largely classical is that the typical classical photon energy

$$
\gamma^{3} / \rho
$$

be small compared with the initial particle energy $\varepsilon=m \gamma$. When, on the contrary,

$$
\Upsilon=\left(\gamma^{3} / \rho\right) / m \gamma=\gamma^{2} /(m \rho)
$$

is large compared with 1 , we have the "ultra quantum" limit, ${ }^{2}$ with the photon typically taking a substantial fraction of the primary energy. For this extreme quantum case, it was pointed out (Ref. 9) that the characteristic length for the convergence of the integrals involved is not $L_{C}$, but the much greater

$$
L_{Q}=\Upsilon^{1 / 3} L_{C}=\Upsilon^{1 / 3} \rho / \gamma=(\gamma / m)^{1 / 3}(\rho / \gamma)^{2 / 3} .
$$

It was suggested therefore that nonuniformity and end effects would be relatively unimportant if the bunch were longitudinally uniform and long, on this scale.

The study of small nonuniformities has been initiated by Pisin Chen, ${ }^{10}$ who 
calculates the corrections proportional to

$$
\left(L_{Q} B^{-1} d B / d z\right)^{2} \text { and } L_{Q}^{2} B^{-1} d^{2} B / d z^{2},
$$

where $B$ is the deflecting force, and $z$ is distance along the particle trajectory. However, we are concerned here with the large nonuniformities that arise at the ends of a region of uniform transverse acceleration, as occur in the beamstrahlung problem with sharply bounded uniform cylindrical space-charge bunches. The quantitative examination of this case has been started by Jacob and $\mathrm{Wu} .{ }^{11-13}$ They have discovered an end effect in the mean energy loss that increases logarithmically with $Y$. We confirm this finding, and calculate also the sublogarithmic terms for large $Y$. The general conclusion is that the end effects are indeed relatively unimportant when the bunch length is large compared with typical values of $L_{Q}$, except in very extreme conditions when even $\ln Y$ is large.

\section{DEPARTURE}

We start with the following formula ${ }^{6}$ for the mean energy $d E$ radiated into a frequency range $d \omega$ and solid angle $d o$ by a relativistic electron:

$$
d E\left[e^{2} \omega^{2} /\left(4 \pi^{2}\right)\right] d \omega d o \int d t \int d t^{\prime} A e^{i \phi},
$$

with

$$
\begin{gathered}
A=\left[\left(\varepsilon^{2}+\varepsilon^{\prime 2}\right) /\left(2 \varepsilon^{\prime 2}\right)\right]\left[v(t) v\left(t^{\prime}\right)-1\right]+(1 / 2)\left(\omega / \varepsilon^{\prime}\right)^{2}(m / \varepsilon)^{2}, \\
\phi=\left(\epsilon / \epsilon^{\prime}\right) \omega\left\{\left(t-t^{\prime}\right)-n\left[r(t)-r\left(t^{\prime}\right)\right]\right\},
\end{gathered}
$$

where $\varepsilon=$ initial electron energy, $\varepsilon^{\prime}=\varepsilon-\omega, r(t)=$ position on classical orbit unperturbed by radiation, $v(t)=(d / d t) r(t), n=$ unit vector in photon direction, $e^{2}=1 / 137$, and $h /(2 \pi)=c=1$.

With

$$
\delta=E / \varepsilon, \quad x=\varepsilon^{\prime} / \varepsilon, \quad(1-x)=\omega / \varepsilon,
$$

the formulae can be rewritten

$$
\begin{gathered}
d \delta=d x d o\left(e^{2} \omega^{2} / 4 \pi^{2}\right) \int d t \int d t^{\prime} A e^{i \phi}, \\
A=\left[\left(1 / 2 x^{2}\right)+(1 / 2)\right]\left[v(t) \cdot v\left(t^{\prime}\right)-1\right]+(1 / 2)[(1-x) / x]^{2} / \gamma^{2}, \\
\phi=(\omega / x)\left(t-t^{\prime}-n \cdot r\right), \\
r=r(t)-r\left(t^{\prime}\right) .
\end{gathered}
$$

Performing the integration over solid angle, i.e., over all directions of $n$, gives

$$
d \delta / d x=\left(e^{2} / \pi\right) \omega x \int d t \int d t^{\prime}(A / r) \sin (\omega r / x) \cos \left[\omega\left(t-t^{\prime}\right) / x\right]
$$


with

$$
r=|r|\left(t-t^{\prime}\right) /\left|t-t^{\prime}\right|
$$

In

$$
\sin (\omega r / x) \cos \left[\omega\left(t-t^{\prime}\right) / x\right]=(1 / 2) \sin \left[\omega\left(r-t+t^{\prime}\right) / x\right]+\sin \left[\omega\left(r+t-t^{\prime}\right) / x\right]
$$

the second term oscillates very quickly for a particle traveling with nearly the velocity of light. Neglecting the variation of $v$ on this scale,

$$
d \delta / d x=-\left(e^{2} / \pi\right) \omega x \int_{-\infty}^{\infty} d t^{\prime}\left[(-\pi / 2) A_{0}+\int_{t^{\prime}}^{\infty} d t(A / r) \sin \theta\right],
$$

where, taking advantage of the symmetry of the integrand, the integral has been restricted to $t>t^{\prime}$, with a compensating factor 2 , and

$$
\theta=(\omega / x)\left(t-t^{\prime}-r\right), \quad A_{0}=-1 /\left(x \gamma^{2}\right) .
$$

We suppose that the unperturbed classical orbit deviates little from a straight line $^{14}$ and drop terms of higher order than second in the deviation. Take Cartesian coordinates with the $z$ axis in the direction of $v\left(t^{\prime}\right)$. Then, for large $\gamma$ and small deviations

$$
\begin{gathered}
v\left(t^{\prime}\right)=\left[0,0,1-1 /\left(2 \gamma^{2}\right)\right], \\
v(t)=\left[v_{x}, v_{y}, 1-1 /\left(2 \gamma^{2}\right)-\left(v_{x}^{2}+v_{y}^{2}\right) / 2\right], \\
1-v(t) \cdot v\left(t^{\prime}\right)=1 / \gamma^{2}+\left(v_{x}^{2}+v_{y}^{2}\right) / 2, \\
r=\left(z^{2}+x^{2}+y^{2}\right)^{1 / 2}=z+(1 / 2)\left(x^{2}+y^{2}\right) / z, \\
(r-t)=(z-t)+(1 / 2)\left(x^{2}+y^{2}\right) /\left(t-t^{\prime}\right) .
\end{gathered}
$$

Then

$$
\begin{gathered}
A=-\left[\left(1+x^{2}\right) /\left(2 x^{2}\right)\right]\left[1 / \gamma^{2}+\left(v_{x}^{2}+v_{y}^{2}\right) / 2\right]+(1-x)^{2} /\left(2 x^{2} \gamma^{2}\right), \\
\theta=\psi-(1 / 2)(\omega / x)\left(x^{2}+y^{2}\right) /\left(t-t^{\prime}\right), \\
\partial \psi / \partial t=(\omega / x)\left[1 /\left(2 \gamma^{2}\right)+\left(v_{x}^{2}+v_{y}^{2}\right) / 2\right], \quad \psi\left(t^{\prime}\right)=0,
\end{gathered}
$$

(where $x$ is used in two different senses!).

It is convenient to introduce normalized variables, denoted by capital letters, such that

$$
\begin{gathered}
t=(\rho / \gamma) T, \\
v_{x}=V_{x} / \gamma, \quad v_{y}=V_{y} / \gamma, \\
(x, y)=(\rho / \gamma)(X, Y) / \gamma,
\end{gathered}
$$

where $\rho$ is some typical radius of curvature. Then

$$
d \delta / d x=\left(e^{2} / \pi\right) \Omega \int d T^{\prime}\left\{\left[\int d T(F / \tau) \sin \theta\right]-(\pi / 2) F_{0}\right\}
$$




$$
\begin{gathered}
T>T^{\prime} \\
\tau=T-T^{\prime}, \\
F=\left[1+\left(V_{x}^{2}+V_{y}^{2}\right) / 2\right]\left(1+x^{2}\right)-(1-x)^{2}, \quad F_{0}=2 x, \\
\theta=\psi-\Omega\left(X^{2}+Y^{2}\right) / \tau, \\
\partial \psi / \partial T=\Omega\left(1+V_{x}^{2}+V_{y}^{2}\right), \\
\Omega=(1-x) /(2 x Y), \quad Y=\left(\gamma^{2} / m \rho\right) .
\end{gathered}
$$

The scaling variable $Y$ is essentially that of Noble. ${ }^{8}$ It measures the ratio of the typical photon frequency of the classical case, of order $\gamma^{3} / \rho$, to that typical in the quantum case, of order $m \gamma$. When $\Gamma$ is large, quantum effects are essential. We use the terminology

$$
\begin{gathered}
Y \gg 1, \quad \text { "extreme quantum" } \\
Y \ll 1, \quad \text { "classical". }
\end{gathered}
$$

It remains to write equations of motion for $X, Y, V_{x}, V_{y}$ :

$$
\begin{gathered}
d X / d T=V_{x}, \quad d V_{x} / d T=K_{x} \\
d Y / d T=V_{y}, \quad d V_{y} / d T=K_{y} \\
\left(X, Y, V_{x}, V_{y}\right)=0 \quad \text { at } T=T^{\prime} .
\end{gathered}
$$

The quantity $K$, in general a function of $T$, is the deflecting force in units of that defining the typical radius of curvature $\rho$. In the present paper we will consider only a deflecting force uniform over a segment of the trajectory:

$$
\begin{aligned}
& K_{x}=0 \\
K_{y}=0 & \text { outside the field } \\
=1 & \text { inside the field. }
\end{aligned}
$$

\section{CENTRAL EFFECT}

For a sufficiently long field, and for $T^{\prime}$ sufficiently far from the ends,

$$
\begin{gathered}
\left(d^{2} \delta / d x d T^{\prime}\right)=\left(e^{2} / \pi\right) \Omega\left[\int_{0}^{\infty}(d \tau / \tau) F \sin \Omega\left(\tau+\tau^{3} / 12\right)-(\pi / 2) F_{0}\right] \\
F=\left(1+\tau^{2} / 2\right)\left(1+x^{2}\right)-(1-x)^{2}, \quad F_{0}=2 x .
\end{gathered}
$$

This gives, in terms of Airy functions,

$$
\left(d^{2} \delta / d x d T^{\prime}\right)=-e^{2}(1-x) /(x Y)\left[\left(1+x^{2}\right) A i^{\prime}(f) / f+x \int_{f}^{\infty} d v A i(v)\right],
$$

with

$$
f=[(1-x) /(x Y)]^{2 / 3}
$$


This is one form of the result of Sokolov and Ternov (Ref. 15, Eq. 8). As pointed out by them, it simplifies for large $Y$ :

$$
\left(d^{2} \delta / d x d T^{\prime}\right)=\left(e^{2} / \pi\right)[(1-x) /(x Y)]^{1 / 3}\left(1+x^{2}\right)\left(-\pi A i^{\prime}(0)\right),
$$

where

$$
-\pi A i^{\prime}(0)=0.813
$$

One comes directly to the asymptotic form [Eq. (39)] by noting in Eq. (35) that the integral converges when the phase oscillates rapidly. For large $Y$, and so large $\Omega$ (for $x$ not very small), this happens only at large $\tau$. The $\tau^{3}$ term then dominates in the phase, and the $\tau^{2}$ in $F$. Retaining only these gives Eq. (39).

More precisely, remembering that our unit of time, or distance, is $L_{C}=\rho / \gamma$, we see that in the extreme quantum case the characteristic time, or length, at which rapid oscillation sets in is of order

$$
L_{Q x}=(2 \Omega)^{-1 / 3} \rho / \gamma=(x Y /(1-x))^{1 / 3}(\rho / \gamma),
$$

which for $x$ not very small or large is of order $L_{Q}$.

Integrating Eq. (39) over $x$, we have in the central region

$$
d \delta / d t=1.165\left(e^{2} / \pi\right) L_{Q}^{-1}=1.165\left(e^{2} / \pi\right)\left(m \gamma / \rho^{2}\right)^{1 / 3},
$$

where $t$ is time in ordinary units, in whatever reference frame is used.

For application to the beamstrahlung problem, with a uniform cylindrical bunch and small disruption, a further integration is required, over impact parameter $b$. Since $\rho^{-1}$ is proportional to $b$, and therefore $L_{Q}^{-1}$ to $\left(b^{2}\right)^{1 / 3}$, the appropriate average over $b^{2}$ gives a factor $3 / 4$. The average fractional energy loss for a particle moving axially through a uniform cylindrical bunch is then

$$
d \delta / d t=0.874\left(e^{2} / \pi\right) L_{\mathrm{QB}}^{-1}
$$

where $L_{\mathrm{QB}}$ is just $L_{\mathrm{Q}}$ evaluated at maximum impact parameter $\mathrm{B}$. Note that if the laboratory frame of reference is used, the transit time is bunch length $/ 2$, and it is this by which Eq. (42) must be multiplied to get the integrated central effect. Following Himel and Siegrist ${ }^{7}$ formulae such as Eq. (42) were given by several authors. We checked against that of Blankenbecler and Drell. ${ }^{16}$

\section{END EFFECTS}

Near the ends we have to be more careful. Let us divide the ranges of $T^{\prime}$ and $T$ in Eq. (24) into three parts, 1,2, and 3, corresponding to before, during, and after the application of the field. Then the integral can be written with an obvious notation

$$
I_{11}+I_{12}+I_{13}+I_{22}+I_{23}+I_{33} \text {. }
$$

We can evaluate $I_{11}$ from Eq. (24), if we are careful with the convergence of the integral at infinity, by introducing, for example, a convergence factor

$$
e^{-\varepsilon|T|} e^{-\varepsilon\left|T^{\prime}\right|}
$$


where $\varepsilon$ is very small. It is simpler to go back to Eq. (9):

$$
\begin{aligned}
I_{11}= & \left(e^{2} \omega^{2}\right) /\left(8 \pi^{2} x^{2}\right)\left[\left(1+x^{2}\right)\left(v^{2}-1\right)+(1-x)^{2} / \gamma^{2}\right] \\
& \times\left.\left.\int d o\right|_{-\infty} \int^{0} d t \exp [i(\omega / x)(1-n \cdot v) t]\right|^{2} \\
= & -\left(e^{2} x\right) /\left(4 \pi^{2} \gamma^{2}\right) \int d o(1-n \cdot v)^{-2}
\end{aligned}
$$

or

$$
I_{11}=-\left(e^{2} / \pi\right) x
$$

For the next integral we go to Eq. (24) (leaving the $\pi / 2$ term to $I_{11}, I_{22}$, and $I_{33}$ )

$$
I_{12}=\left(e^{2} / \pi\right) \Omega \int_{-\infty}^{0} d T^{\prime} \int_{0}^{\infty} d T(F / \tau) \sin \theta,
$$

where

$$
\begin{gathered}
\tau=T-T^{\prime} \\
F=\left(1+T^{2} / 2\right)\left(1+x^{2}\right)-(1-x)^{2} \\
\theta=\Omega\left[T-T^{\prime}+T^{3} / 3-(1 / 4) T^{4} / \tau\right] .
\end{gathered}
$$

This is some function of $\Omega$, which we will calculate analytically only for $\Omega$ small. The $T^{2}$ term in $F$ then dominates the other (by a factor of order $\Omega^{-2 / 3}$ ), and we have

$$
I_{12}=\left(e^{2} / 2 \pi\right)\left(1+x^{2}\right)(A+B)
$$

where

$$
\begin{gathered}
A=\Omega \int_{0}^{\infty} d \tau / \tau \int_{0}^{\tau} d T T^{2} \sin \left[\Omega\left(\tau+T^{3} / 3\right)\right] \\
B=\Omega \int_{0}^{\infty} d \tau / \tau \int_{0}^{\tau} d T T^{2}\left\{\sin \left[\Omega\left(\tau+T^{3} / 3-T^{4} / 4 \tau\right)\right]-\sin \left[\Omega\left(\tau+T^{3} / 3\right)\right]\right\}
\end{gathered}
$$

In the following sequence we drop at each stage terms that vanish for small $\Omega$; the " $=$ " sign should be qualified accordingly. The integration range near $\tau=0$ has to be treated with care because of the factor $(1 / \tau)$. Then

$$
\begin{aligned}
A & =\int_{0}^{\infty}(d \tau / \tau)\left\{\cos (\Omega \tau)-\cos \left[\Omega\left(\tau+\tau^{3} / 3\right)\right]\right\} \\
& =\int_{0}^{1}(d \tau / \tau)\left[\cos \tau-\cos \left(\tau+\Omega^{-2} \tau^{3} / 3\right)\right]+\int_{1}^{\infty}(d \tau / \tau) \cos \tau \\
& =\int_{0}^{1}(d \tau / \tau) \cos \tau\left[1-\cos \left(\Omega^{-2} \tau^{3} / 3\right)\right]+\int_{1}^{\infty}(d \tau / \tau) \cos \tau \\
& =\int_{0}^{1}(d \tau / \tau)\left[1-\cos \left(\Omega^{-2} \tau^{3} / 3\right)\right]+\int_{1}^{\infty}(d \tau / \tau) \cos \tau
\end{aligned}
$$




$$
\begin{aligned}
& +\int_{0}^{1}(d \tau / \tau)(\cos \tau-1)\left[1-\cos \left(\Omega^{-2} \tau^{3} / 3\right)\right] \\
= & (1 / 3) \int_{0}^{G}(d \tau / \tau)(1-\cos \tau)+\int_{1}^{\infty}(d \tau / \tau) \cos \tau+\int_{0}^{1}(d \tau / \tau)(\cos \tau-1)
\end{aligned}
$$

(where $G=\Omega^{-2} / 3$ )

$$
\begin{aligned}
= & (1 / 3) \int_{1}^{G}(d \tau / \tau)(1-\cos \tau)+\int_{1}^{\infty}(d \tau / \tau) \cos \tau+(2 / 3) \int_{0}^{1}(d \tau / \tau)(\cos \tau-1) \\
= & (2 / 3) \ln \Omega^{-1}-(1 / 3) \ln 3+(2 / 3) \int_{1}^{\infty}(d \tau / \tau) \cos \tau \\
& +(2 / 3) \int_{0}^{1}(d \tau / \tau)(\cos \tau-1) \\
= & (2 / 3) \ln \Omega^{-1}-(1 / 3) \ln 3-(2 / 3) \gamma
\end{aligned}
$$

(where $\gamma$ is Euler's constant)

$$
=(2 / 3)\left(\ln \Omega^{-1}-1.127\right) \text {. }
$$

In the integral $B$, it is convenient to replace $T$ by $\chi=T / \tau$ :

$$
\begin{aligned}
B & =\Omega \int_{0}^{\infty} d \tau \tau^{2} \int_{0}^{1} d \chi \chi^{2}\left[\sin \left(\Omega\left\{\tau+\left(\tau^{3} / 3\right)\left[\chi^{3}-(3 / 4) \chi^{4}\right]\right\}\right)-\sin \left[\Omega\left(\tau+\tau^{3} \chi^{3} / 3\right)\right]\right] \\
& =\int_{0}^{\infty} d \tau \tau^{2} \int_{0}^{1} d \chi \chi^{2} \\
& \quad\left[\sin \left\{\Omega^{2 / 3} \tau+(1 / 3) \tau^{3} \chi^{3}[1-(3 / 4) \chi]\right\}-\sin \left[\Omega^{2 / 3} \tau+(1 / 3) \tau^{3} \chi^{3}\right]\right]
\end{aligned}
$$

We now put $\Omega=0$ and do the $\tau$ integration. Then

$$
\begin{aligned}
B & =\int_{0}^{1}(d \chi / \chi)\left\{[1-(3 / 4) \chi]^{-1}-1\right\} \\
& =\int_{0}^{1} d \chi(3 / 4)[1-(3 / 4) \chi]^{-1}=\ln 4 \\
& =1.386 .
\end{aligned}
$$

So

$$
I_{12}=\left(e^{2} / \pi\right)\left(1+x^{2}\right)\left[(1 / 3) \ln \Omega^{-1}+0.3176\right],
$$

where ${ }^{17}$ we have taken (with $\gamma$ denoting Euler's constant)

$$
(1 / 2) \ln 4-(1 / 6) \ln 3-\gamma / 3=0.3176 .
$$

The integral $I_{13}$, if the field region is long compared with $L_{\Omega}=\Omega^{-1 / 3}(\rho / \gamma)$, will involve the sine of a large phase, which will oscillate rapidly with the parameters defining the situation. We can take then

$$
I_{13}=0
$$


For $I_{22}$, when the field is sufficiently long, the main contribution is the first part of

$$
I_{22}=I_{220}+\Delta I_{22}
$$

where

$$
\begin{gathered}
I_{220}=\left(e^{2} / \pi\right) \Omega \int_{-L}^{0} d T^{\prime}\left(\left\{\int_{T^{\prime}}^{\infty} d T(F / \tau) \sin \left[\Omega\left(\tau+\tau^{3} / 12\right)\right]-F_{0} \pi / 2\right\}\right), \\
\Delta I_{22}=-\left(e^{2} / \pi\right) \Omega \int_{-L}^{0} d T^{\prime} \int_{0}^{\infty} d T(F / \tau) \sin \left[\Omega\left(\tau+\tau^{3} / 12\right)\right] .
\end{gathered}
$$

The first term is just the central effect [Eq. (42)] multiplied by field length $L$. The second term is the correction for having taken the $T$ integral to infinity, rather than 0 , in the first (we have shifted the origin of $T^{\prime}$ and $T$ to the exit point). For $L$ large enough, we can replace $-L$ as lower limit of $T^{\prime}$ in $I_{220}$ by $-\infty$. Then

$$
\begin{aligned}
\Delta I_{22} & =-\left(e^{2} / \pi\right) \Omega \int_{-\infty}^{0} d T^{\prime} \int_{-T^{\prime}}^{\infty} d \tau(F / \tau) \sin \left[\Omega\left(\tau+\tau^{3} / 12\right)\right] \\
& =-\left(e^{2} / \pi\right) \Omega \int_{0}^{\infty} d \tau F \sin \left[\Omega\left(\tau+\tau^{3} / 12\right)\right] .
\end{aligned}
$$

For small $\Omega$, taking

$$
F=(1 / 2) \tau^{2}\left(1+x^{2}\right)
$$

we have, absorbing $\Omega^{1 / 3}$ into the integration variable,

$$
\begin{aligned}
\Delta I_{22} & =-\left(e^{2} / 2 \pi\right)\left(1+x^{2}\right) \int_{0}^{\infty} d \tau \tau^{2} \sin \left[\tau^{3} / 12\right] \\
& =-\left(e^{2} / 2 \pi\right) 4\left(1+x^{2}\right) \int_{0}^{\infty} d \tau \sin \tau \\
& =-2\left(e^{2} / \pi\right)\left(1+x^{2}\right) .
\end{aligned}
$$

The integral $I_{23}$ looks different from $I_{12}$ only because of our unsymmetric treatment of $T$ and $T^{\prime}$. We have

$$
I_{23}=\left(e^{2} / \pi\right) \Omega^{-1} \int_{-\infty}^{0} d T^{\prime} \int_{0}^{\infty} d T(F / \tau) \sin \theta
$$

From Eqs. (33) and (34) in the range of the integral

$$
\begin{gathered}
V_{Y}=+T^{\prime} \\
Y=-(1 / 2) T^{\prime 2}+T^{\prime} T \\
\theta=\Omega\left[-T^{\prime}-(1 / 3) T^{\prime 3}+T\left(1+T^{\prime 2}\right)-Y^{2} /\left(T-T^{\prime}\right)\right] \\
=\Omega\left[T-T^{\prime}-(1 / 3) T^{\prime 3}-(1 / 4) T^{\prime 4} /\left(T-T^{\prime}\right)\right] .
\end{gathered}
$$

Then with a change of variables replacing $T^{\prime}$ and $T$ by $-T$ and $-T^{\prime}$, respectively, 
and comparing with Eqs. (47) and (48),

$$
I_{23}=I_{12} \text {. }
$$

Finally,

$$
I_{33}=I_{11} \text {. }
$$

Collecting terms, the total and effect is

$$
\begin{aligned}
(d \delta / d x)_{\mathrm{end}} & =2 I_{11}+2 I_{12}+\Delta I_{22} \\
& =\left(e^{2} / \pi\right)\left[-2 x+\left(1+x^{2}\right)\left((2 / 3) \ln \Omega^{-1}-1.365\right)\right] \\
& =\left(e^{2} / \pi\right)\left[(2 / 3)\left(1+x^{2}\right)\left(\ln \Omega^{-1}-2.047\right)-2 x\right] .
\end{aligned}
$$

Integrating over $x$ [assuming $Y$ large enough that $x Y /(1-x)$ is large over most of the range]

$$
\delta_{\text {end }}=\left(e^{2} / \pi\right)(8 / 9)(\ln Y-2.104) .
$$

In the case of a circular cylinder of uniform space charge, the deflecting field increases linearly with radius, and so then does $Y$, to a maximum value $Y_{B}$. Then the appropriate average is

$$
\delta_{\text {end }}=\left(e^{2} / \pi\right)(8 / 9)\left(\ln Y_{B}-2.604\right) .
$$

This last case is that considered by Jacob and $\mathrm{Wu} .{ }^{11-13}$ The logarithmic rise with very large $Y$ is their discovery.

Comparing Eq. (72) with the central effect [Eq. (39)], the effective increase in field length is

$$
\Delta L=0.820\left[\ln \Omega^{-1}-2.047-3 x /\left(1+x^{2}\right)\right] L_{Q x}
$$

with

$$
L_{Q x}=[x Y /(1-x)]^{1 / 3}(\rho / \gamma), \quad \Omega=(1-x) /(2 x Y) .
$$

Comparing Eq. (41) with Eq. (73) integrated over the photon spectrum, the end effect corresponds to an increase in effective length

$$
\Delta L=0.763 L_{\mathrm{Q}}(\ln Y-2.104) \text {. }
$$

Finally, comparing Eq. (42) with Eq. (74), averaged over the impact parameter for a uniformly charged cylinder, the effective increase in length is

$$
\Delta L=1.017\left(\ln Y_{B}-2.604\right) L_{Q B},
$$

where subscript $B$ indicates evaluation at the beam edge.

\section{CONCLUSION}

Our main result is Eq. (72). We used it here to discuss only mean energy loss. Clearly we could go on to discuss photon numbers and fluctuations, following Noble $^{8}$ and Yokoya. ${ }^{18}$ 
It is natural to suppose that the "end effects" actually occur at the ends. Strictly speaking, in this approach only the total integral is significant, however convenient it may be to separate out (in a gauge-dependent way) different contributions in the calculation. It would be good to see an approach that explicitly keeps track of the approximate space-time development.

\section{ACKNOWLEDGMENTS}

We profited greatly from the 1986 CLIC meetings on this topic. In particular it was the justified scepticism there of W. Willis, in the face of careless statements by one of us, that led to recognition of the role of $L_{Q}$. We are indebted also to Pisin Chen and S. D. Drell, and especially to M. Jacob and T. T. Wu, for very useful discussions.

\section{REFERENCES}

1. J. Schwinger, Proc. Nat. Acad. Sci. 40, 132 '1954).

2. A. A. Sokolov and I. M. Ternov, Synchrotron Radiation (Pergamon 1971).

3. A. N. Matveev, ZETF 31, 479 (1956); Sov. Phys. JETP 4, 409 (1957).

4. A. I. Nikishov and V. I. Ritus, Sov. Phys. JETP 25, 1135 (1967).

5. V. N. Baier and V. M. Katkov, Sov. Phys. JETP 28, 807 (1969), and Sov. Phys. JETP 26, 854 (1968).

6. V. B. Berestetskii, E. M. Lifshitz, and L. P. Pitaevskii, Relativistic Quantum Theory (Pergamon 1971).

7. T. Himel and J. Siegrist, SLAC-PUB-3572 (Feb. 1985).

8. R. J. Noble, SLAC AAS-Note 3 (Dec. 1985).

9. J. S. Bell, CLIC meeting, Sep. 9, 1986.

10. Pisin Chen, SLAC-PUB-4391 (Aug. 1987).

11. M. Jacob and T. T. Wu, Phys. Lett. 197B, 253 (1987).

12. M. Jacob and T. T. Wu, CERN-TH.4848/87.

13. M. Jacob and T. T. Wu, CERN-TH.4907/87.

14. It suffices that this be true over distances required for the convergence of the integral, of order $L_{Q}$ in the ultraquantum case.

15. M. Bell and J. S. Bell, CERN PS 87-53(DL), June 1987; Particle Accelerators, in press.

16. R. Blankenbecler and S. D. Drell, Phys. Rev. B36, 277 (1987).

17. When these results were presented at the CLIC meeting of Nov. 13, 1987, this constant was approximated by 0.25 , as a result of a rough numerical integration. The integrals involved in defining the constants in this and the following formulae have now been done analytically.

18. K. Yokoya, Nuclear Instruments A251, 1 (1986). 